مجلة كلية التربية الرياضية - جامعة بغداد * المجلد السابع والعشرون * العدد الرابع * لسنة

\title{
دراسة تحليلية لأداء المهارات الهجومية وعلاقتها بترتيب الفرق في الكرة الطائرة للنساء
}

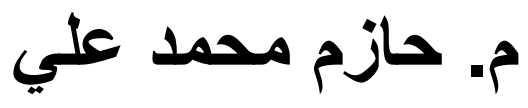

\section{Abstract.}

\section{Analytical study of the performance of the offensive skills and their relationship to the difference in the order of women's volleyball.}

The research covered the five-door:

Chapter One: contains the door on the front and the importance of research and touched on the volleyball game and includes the attacking skills, which includes transmitter and battery crushing and wall Alsdomchklh search in the analysis of attacking skills (transmitter , Aldharbb overwhelming, bulwark) with the order of teams participating in the League women's volleyball $r \cdot 1 r$.

Part II: This has touched the door to Alndharah studies related to the subject of the study included the terms of the basic skills in the game of volleyball offensive and defensive, and the importance of analysis in the game, as well as previous studies.

Part III: research methodology and procedures Field

Then addressed in this section to how to test the research methodology, the researcher used the descriptive approach style Relations connectivity to Mlomth with the nature of the research and how to choose the sample and Cat sample of four teams and the League for the two phases and the means to gather information and the tools and equipment used as well as steps to conduct research and how to design a form of observation and evaluation, and a main experiment as well as statistical methods.

Part IV: Results , analysis and discussion

This included the door to view and analyze and discuss their relationship with offensive skills arrange league teams as well as the differences in the performance of the offensive skills and their relationship to arrange league teams. If Adhrt results that there is a relationship between random and arranging skills offensive teams in the league for the women's volleyball season in $r \cdot 1 r$ and has been discussing these results in a scientific manner supported by scientific sources.

Part V: Conclusions and recommendations 
This included the door on the conclusions drawn by the researcher of the study and during the most important links in a random percentage of the level of performance of the offensive skills. The highest percentage of successful attempts were beating overwhelming skill

Recommendations

1. Necessary to work to diversify the offensive skills to fit the level of the defending team to increase the impact of attack

$r$. Bulwark focus on finding more of the player have the ability to master the skill bulwark.

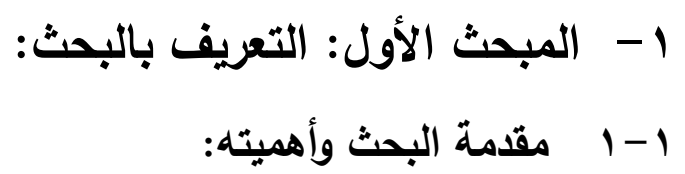

النطورات العلمية التي اجتاحت المجالات كافة في العالم كان للمجال الرياضي، حصة كبيرة منها والذي يتطلب إجراء بحوث ودراسات عديدة من أجل التعرف على حل المشكلات التي تعاني منها.

ومما لاشك ان لعبة الكرة الطائرة تشمل على مهارات دفاعية واخرى هجومية اذان المهارات الهجومية هي

(الارسال والضرب الساحق وحائط الصد الهجومي) ومهارة الضرب الساحق هو ضرب الكرة بطرق مختلفة من فوق حافة الثبكة نحو ملعب الخصم وبإحدى الذراعين ويعتبر الضرب الساحق هو في مقدمة المهارات في اكتساب نقطة للفريق وكثير من المهارات والخطط تصبح غير مشوقه وحماسية اذ لم تتته بالضرب الساحق ـ لذا نكمن اهمية البحث في تحليل جهد الفرق من خلال المهارات الهجومية وعلاقتها بترتيب الفرق من اجل الحصول على ارقام احصائية ونسب مئوية ووضعها امام الددربين والتركيز عليها من الوحدات التدريبية للوصول الى مستويات عالية في لعبة الكرة الطائرة للنساء.

\section{r-1}

ان الفريق الذي يريد ان يكسب المباراة بالكرة الطائرة عليه استثمار الهجوم الناجح من خلال الدفاع فبدون ذلك تضيع جهود كبيرة بذلت من اللاعبين ومدربيهم فهناك الكثير من الفرق بالكرة الطائرة للنساء تمتاز بدفاع جيد ولكن الهجوم دون المستوى الطموح فيخسر الفريق.

وبما ان المهارات الهجومية الارسال والضرب الساحق وحائط الصد هي الاساس في الهجوم الهنظم للفريق لغرض هجوم جيد لذا ارتئ الباحث دراسة هذه المشكلة والوقوف على المستوى الحقيقي للفرق العراقية للنساء.

$$
\text { r-1 }
$$

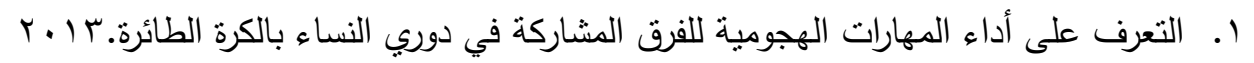
r. التعرف على العلاقة بين المهارات الهجومية مع ترتيب الفرق المشاركة. 
مجلة كلية التربية الرياضية - جامعة بغداد * المجلد السابع والعثرون * العدد الرابع * لسنة

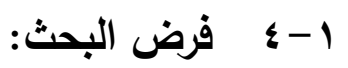

هناك علاقة ذات دلالة إحصائية بين المهارات الهجومية (الإرسال، الضرب الساحق، حائط الصد) مع نرتيب

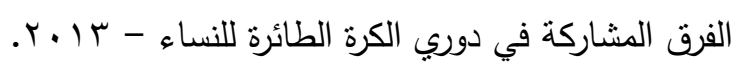

1 - 1 مجالات البحث:

1-ه-1 المجال البشري: يشمل الفرق المشاركة بالدوري وعددهم (ع) فرقهم (أكاد، قرقش، بغداد، شقلاوة) يشمل مباريات فرق الدوري المشاركين في المربع الذهبي وعدد ع للموسم / با • r.

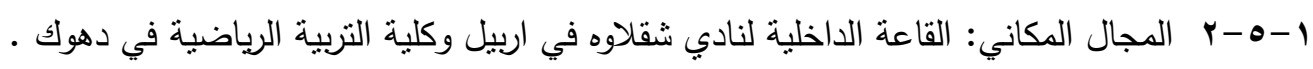

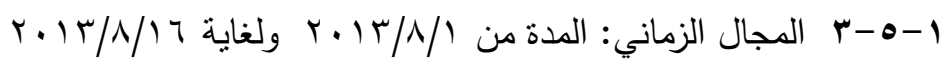

r - r - r الباب الثاني: الاراسات النظرية والسابقة.

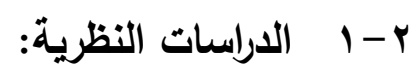

r-1 - 1 المهارات الأساسية لعبة الكرة الطائرة.

لكل لعبة رياضية مهارتها الخاصة بها التي تختلف عن الألعاب الأخرى وفي داخل اللعبة الواحدة هناك اختلاف بين مهارة وأخرى، ولعبة الكرة الطائرة تعد إحدى النماذج الجيدة للألعاب الجماعية التي تزخر بأنواع المهارات الهجومية والدفاعية ، إذ ينوقف إلى حد كبير وصول اللاعب والفريق إلى المستويات العليا عليها ، إذ يجب إتقانها اتقاناً تاما حيث تتفق ((زينب فهمي)) (1:؛ ()) ((حمدي عبد المنعم)) (ץ:rr)على إن المهارات الأساسية تلك الحركات التي يحتاج اللاعب إلى أدائها في الموقف جميعها التي تتطلبها اللعبة للوصول إلى أفضل النتائج مع الاقتصاد التام في المجهود إذا أن فقدت إحدى هذه المهارات أو ضعف المستوى في احدها سيودي إلى خسارة نقاط كثيرة إذا ما أكتشفها

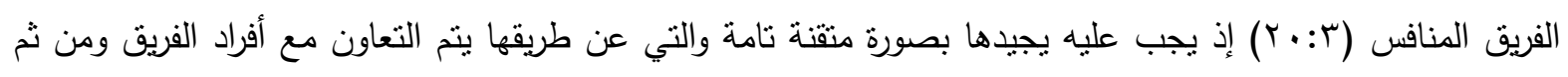
يمكن عن طريق إتقان هذه المهارات تتفيذ الخطط الفنية الموضوعة للهجوم والدفاع.

إذ أن تطور مستوى أي لاعب ومدى نجاحه يعتدد إلى حد كبير على مدى درجة أداء اللاعب المهارة التي

يمكن منها أن يتحقق ذلك من خلال إتباع الأسلوب الصحيح في طرائق ووسائل التعليم والتدريب كما يعرفها عقيل الكاتب (وآخرون) بأنها ((تلك المهارات التي يحتاجها اللاعب عند أدائها في المواقف جميعها التي تتطلبه اللعبة لغرض القيام بالحركة وبأسلوب هادف واقتصادي لتحقيق النتيجة الأفضل والوصول بالرياضي إلى مستوى عال)) (ع:بانها (ب) والكرة الطائرة من الألعاب التي ترتبط قدراتها المهارية الواحدة بالأخرى ارتباطاً وثيقاً وهي ست مهارات (الإرسال، الاستقبال، الإعداد، الضرب الساحق، حائط الصد، الدفاع عن الملعب) وتقسم المهارات الأساسية لكرة الطائرة مهارات هجومية

$$
\text { ودفاعية (0: }
$$


مجلة كلية التربية الرياضية - جامعة بغداد * المجلد السابع والعشرون * العدد الرابع * لسنة

\section{r- المبحث الثالث: منهج البحث وإجراءاته الميدانية. 1-}

استخدام الباحث المنهج الوصفي بالأسلوب المسحي التحليلي الوهو الطريق الذي يعتمد على التقكير الاستقرائي والاستتناجي وتنتخدم اساليب الملاحظة العلمية وفرض الفروض والتجربة لحل مشكلة معينة والوصول الى

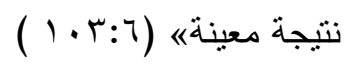

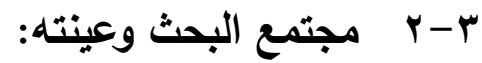

تم اختيار مجتمع البحث بالطريقة العمدية المتنظة بأندية القطر للنساء بالكرة الطائرة با ـ ب البالغ عددهم ( • () ناديا وهم (اكاد، قرقوش، شقلاوة، فتاة النجف، بغداد، الارمني، خاك، سيروان، الوحدة، الزعيم)

تقسم الى مجموعنين كل مجموعة (0) انديه وكان المجموعة الاولى هم (أكاد، قرقوش، فتاة النجف، الوحدة، خاك) واما المجموعة الثانية كانت كل من (ثقلاوة، بغداد، الارمني، سيروان، الزعيم) تجري لمرحلة واحده يخرج فرقين من كل مجموعة والتي اقيمت في قاعة الداخلية لنادي شقلاوة في اربيل ترشح من المجموعة الاولى كل من نادي (أكاد وقرقوش) والمجموعة الثانية كان (شقلاوة وبغداد) حيث تجري المباراة على مرحلتين. وتعد عينة البحث ضرورة من ضروريات البحث العلمي وهي " جزء من المجتمع تقوم بدراسته للتعرف على (00:V) (مصائص المجتمع الذب سحبت منه العينة) أختار الباحث العينة بطريقة العدية. لكون أندية الأربعة المترشحة تمثل أعلى مستوى رياضياً بين الأندية العراقية ويلعب كل فريق (ץ) مباريات أي مجموع المباريات (†) مباراة عن طريق الدوري لمرحلتين وتم أخذ المباريات

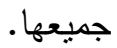

\section{r-r وسائل جمع المطلومات والأدوات والأجهزة:}

من أجل الحصول على حقائق صحيحة ومتكاملة لحل المشكلات فقد استعان الباحث بأدوات عديدة

$$
\begin{aligned}
& \text { للحصول على المعلومات والبيانات المطلوبة وهي: } \\
& \text { 1. المراجع والمصادر العربية والأجنبية. } \\
& \text { r. استمارة خاصة للمسح الميداني. } \\
& \text { r. التجربة الاستطلاعية. }
\end{aligned}
$$


ع. فريق عمل لملاحظة المباريات("). 0. التصوير بجهاز الفيديو وتحليل الأفلام. 7. المباراة الرسمية لعينة البحث.

\section{r-s خطوات أجراء البحث:}

r- צ- 1 تصميم استمارة الملاحظة وتقويمها: بعد إطـلاع الباحث على بعض المصـادر والمراجـع والبحوث والدراسـات المتوفرة والتي تخص لعبـة كرة الطائرة، ومن اجل اجراء عملية التقويم بصورة دقيقة فقد اعتمد الباحث على استمارة خاصة لتقويم مستوى اداء المهارات الهجومية في الكرة الطائرة، قام الباحث بتعديلها بمحاور جديدة وهي مأخوذة من رسالة خليل ستار (^:؟ 7)

\section{r- r- r التجرية الاستطلاعية:}

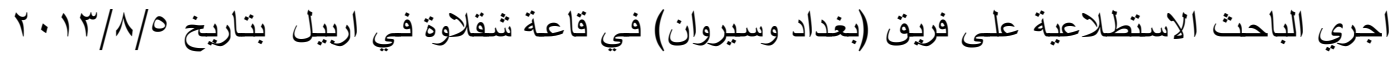
لغرض معرفة المتغيرات جميعها التي تؤثر في كيفية عمل الاستمارة وكفاءة الكادر المساعد وإمكانيتهم من ملاحظة المهارات الهجومية والنقاط المسجلة من خلالها وتسجيلها في الاستمارة المعدة لهذا الغرض والتعرف على نقاط الضعف فيها لإجراء التعديلات وتصحيح الأخطاء التي يمكن أن يقع الباحث وفريق العمل فيها.

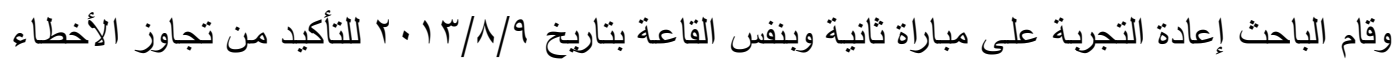

\section{r-ع - ب الأسس العلمية للاستمارة:

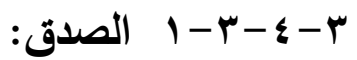

إن صـدق الاختبـار يعني: ان الاختبـار يقيس مـا اعد لقياسـه. ( 9: .ب ا) ولغرض النأكد مـن صـق

الاستمارة المعدة لتحليل بالكرة الطائرة لجأ الباحث إلى إيجاد صدق المحتوى وهو قياس تمثيل الاختبار لنواحي الباحث الجانب المقاس بحثه وتحليل مواد الاختبار وعناصر تحليلاً منطقياً لتحديد الوظائف والجوانب الممتلكة فيه نسبة كل

" لقد تكون فريق العمل من المتخصصين والمتداولين للعبة الكرة الطائرة وأساتذة والحاصلين على شهادة الماجستير والبكالوريوس وهم السادة:

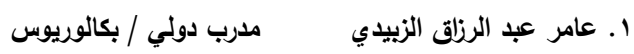

ماجستير تربية رياضية / كلية الادارة والاقتصاد r اياد علي حسين

ماجستير تريية رياضية/ كلية التربية الرياضية r. م. م.م ماهر عبد الإله مدرب منتخب الوطني للنساء سابقا ؛ . حسين عدنان * انظر الملحق رقم (1) 
منها إلى الاختبار بأكمله (^:^§)، وقام الباحث بإيجاد صدق محتوى الاستمارة بتوزيعها على مجموعة من الخبراء والمتخصصين** بالكرة الطائرة لإبداء أراءهم حول ما تحتويه فقرات الاستمارة واتفق الخبراء والمختصون على صدق محتوى الاستمارة في تحقيق الغرض الذي وضعت من اجله ، وبهذا حصل الباحث على صدق محتوى الاستمارة.

$$
\text { : }
$$

الثبات تعبير عن وجود العمل ، ويقصد بثبات الاختبار درجـة التقة ، وذلك لان الاختبار لا يتغير في النتيجة أي ذو قيمة ثابتة خلال التكرار أو الإعادة ( • (: ^^) واستخدام الباحث طريقة إعادة الاختبار لإيجاد معامل ثبات الاختبار ، إذ أعطيت استمارة التحليل إلى أحد الخبراء وطبقت على إحدى مباريات الدوري لأندية القطر للنساء للمرحلة الأولى من التصفيات والتي تم استبعادها من التجربة الرئيسية ثم أعيدت استمارة التحليل إلى الخبير نفسه بعد بـ أيام وقام بتحليل المباراة نفسها ومن ثم حساب معامل الارتباط البسيط بيرسون بين الملاحظة الاولى والملاحظة الثانية والذي على أثزه نوصل الباحث إلى ثبات الاستمارة بعد أن كانت النتيجة ( • 9. •) وهو معامل ارتباط عالٍ.

\section{r-ع}

قام الباحث بتصوير المباريات المربع الذهبي بكامل ذلك لزيادة مصداقية فريق العمل والاستمارة المعدة لهذا

r-ع - تهليل المباريات باستخدام الاستمارة:

قام الباحث بتحليل المباريات من خلال مشـاهدتها بعد التصوير والمقارنة مع ما تم انجازه من فريق العمل

للتأكيد من دقة العمل وزيادة المصداقية.

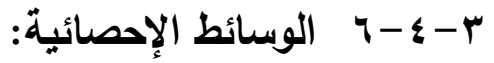

استخدام الباحث البرنامج الإحصائي (Spss) للحصول على النتائج الإحصائية:-

$$
\begin{aligned}
& \text { 1. الوسط الحسابي. } \\
& \text { r. الانحراف المعياري. } \\
& \text { r. النسبة المئوية. } \\
& \text { ع. معامل الارتباط البسيط (بيرسون). } \\
& \text { ه. معامل الارتباط (الرتب) سبيرمان. }
\end{aligned}
$$

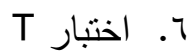


مجلة كلية التربية الرياضية - جامعة بغداد * المجلا السابع والعشرون * العدد الرابع * لسنة

ع - المبحث الرابع: عرض النتائج وتحليلها ومناقشتها. ع - 1 عرض نتائج الأوساط الحسابية والانحرافات المعيارية لمستوى أداء المهارات الهجومية لفرق عينة البحث:

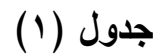

\begin{tabular}{|c|c|c|c|c|c|c|c|}
\hline \multicolumn{2}{|c|}{ حائط الصد } & \multicolumn{2}{|c|}{ الضرب الساحق } & \multicolumn{2}{|c|}{ الإرسال } & الترتيب & الفرق \\
\hline$\varepsilon$ & س س & $\varepsilon$ & س س & $\varepsilon$ & س س & & \\
\hline$\cdot, r \wedge r$ & $r, \Sigma)$ & אדדו, & $r, V Y$ &.,$O V Y$ & $r, 1 \wedge$ & & \\
\hline$\cdot, \varepsilon \cdot V$ & $r, Y_{1}$ & $\cdot, \wedge \leq$. & r,Ar & oro, & $r, I V$ & الثاني & ق ق قرقوش \\
\hline$\cdot, 7 \leq \leqslant$ & $r, \leqslant 0$ & $\cdot, T \vee T$ & $\uparrow, \uparrow \wedge$ &.,$O \wedge Y$ & $r, Y_{1}$ & 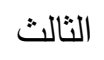 & بغداد \\
\hline$\cdot, Y q \wedge$ & $Y, Y V$ & $\cdot, \vee \vee$. & $Y, V Y$ & ., & r, & الرابع & شقلاوة \\
\hline
\end{tabular}

ع - r ع عرض نتائج علاقة الارتباط النسبية لمستوى أداء المهارات الهجومية وترتيب الفرق في

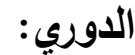

جدول (r)

\begin{tabular}{|c|c|c|c|c|}
\hline الالالة & القيمة الجدلية & قيمة T دلالة معنوية & معامل الارتباط & المهارة \\
\hline عشوائي & $\cdot r, \nabla \wedge$ & $r, \varepsilon r$ & $\cdot, \vee \vee \vee 1$ & الإرسال \\
\hline عشوائي & r,VA & $r_{,} \cdot \varepsilon$ & $\cdot, V \backslash \leq$ & الضرب الساحق \\
\hline عشوائي & r,YA & T, r & $\cdot, 117$ & حائط الصد \\
\hline
\end{tabular}

مـن خـلال الجدول (r) ظهر معامل الارتبـاط لمهارة الارسـال والترتيب لفرق عينـة البحث بقيمـة مقدارها

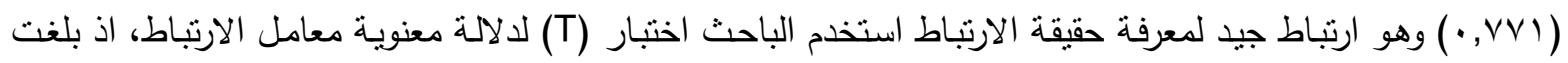

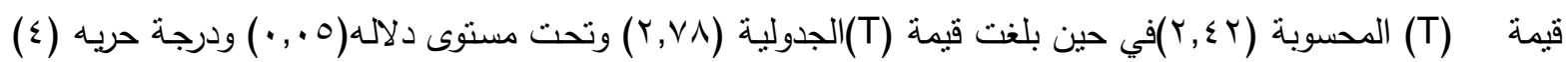
وهي اكبر من قيمة (T) المحسوبة وبذلك يكون الارتباط عشوائيا.

وفي معامل الارتباط لمهارة الضرب الساحق والتزتيب لفرق عينة البحث بقيمة مقدارها (ع (Y, •) وهو ارتباط

جيد ولمعرفة حقيقة الارتباط استخدم الباحث اختبار (T) لدلالة معنوية معامل الارتباط اذ بلغت قيمتها (ع ., ب) في حين 
مجلة كلية التربية الرياضية - جامعة بغداد * المجلد السابع والعشرون * العدد الرابع * لسنة

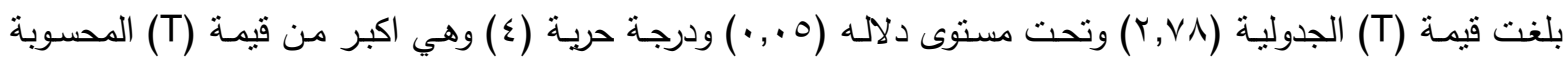
وبذللك يكون الارتباط عشوائيا.

وفي مهارة الحائط الصد معامل الارتباط والترتيب لفرق عينة البحث وقيمة مقدارها (1) (1, •) ولمعرفة حقيقة

الارتباط استخدم الباحث اختبار (T) لدلالة معنوية معامل الارتباط وبلغت قيمتها (TMT, ·) في حين بلغت قيمة (T)

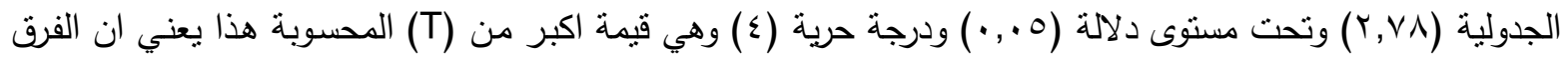

عثوائيا.

ع - r عرض نتائج الأوساط الحسابية والانحرافات المعيارية لنادي أكاد وتحليلها:

الجدول (ץ) - (ب)

يبين الأوساط الحسابية والانحرافات المعيارية لأداء المهارات الهجومية ومجموع المحاولات الكلية لكل مهارة ونسبة للمحاولات الناجحة والفاثلة لنادي أكاد.

\begin{tabular}{|c|c|c|c|c|c|c|c|c|c|c|}
\hline 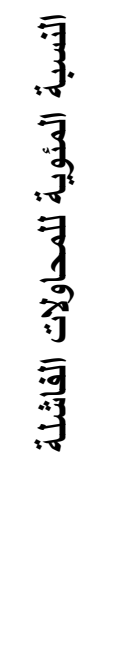 & $\begin{array}{l}3 . \\
3 \\
3 \\
j \\
3 \\
3 \\
\overline{3} \\
3 \\
3 \\
3 \\
\overline{3} \\
\overline{3}\end{array}$ & 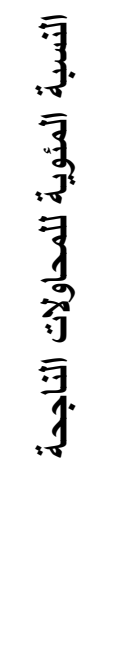 & 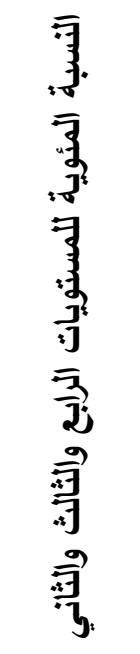 & 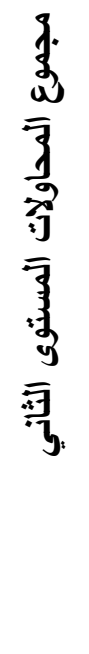 & 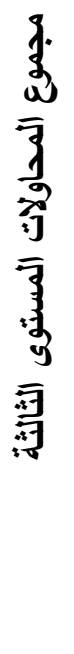 & $\begin{array}{l}\text { j. } \\
3 \\
\overline{3} \\
3 \\
3 \\
\overline{3} \\
\overline{3} \\
3 \\
\overline{3} \\
\overline{3}\end{array}$ & 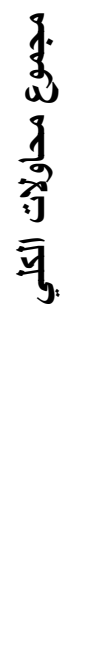 & $\begin{array}{l}\bar{y} \\
\overline{3} \\
\overline{9} \\
\overline{3} \\
3 \\
3 \\
3\end{array}$ & $\begin{array}{l}\overline{3} \\
\overline{3} \\
\overline{3} \\
\overline{3}\end{array}$ & 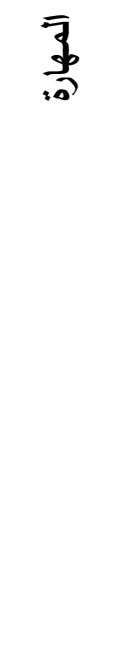 \\
\hline 9 ، Vo & $\varepsilon 1$ & $9.6 \% 0$ & 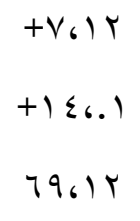 & (9) & 09 & $r$. & $\sum r$ & $\cdot, O V Y$ & $r, 1 \wedge$ & الإرسال \\
\hline $1 \% 690$ & $\vee q$ & $\Lambda V_{6} .0$ & $\begin{array}{l}+\varepsilon .611 \\
+960 . \\
r V_{6}{ }^{2} V\end{array}$ & TYA & 01 & $r \leqslant 0$ & 71. & אדד, • & T.VT & الضرب الساحق \\
\hline . & 79 & $\pi$ Tro. & $\begin{array}{l}+r 961 . \\
+1960 V \\
1 \varepsilon_{6} 11\end{array}$ & rᄉ & TV & 00 & 119 & $\cdot, \Gamma \wedge r$ & $r_{6} \leqslant 1$ & حائط \\
\hline
\end{tabular}


بين الجدول (r) الأوساط الحسابية والانحرافات المعيارية لأداء المهارات الهجومية الإرسال، حائط الصد،

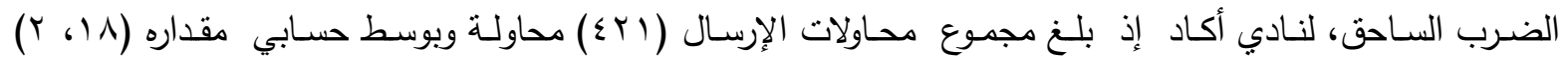

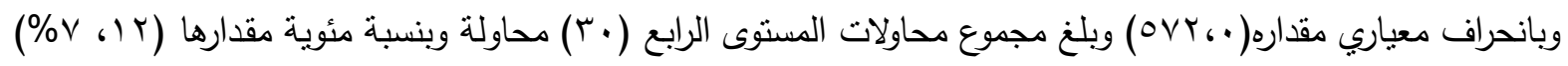

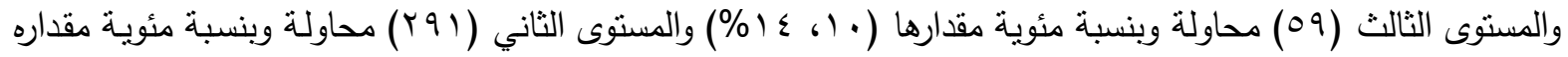
(r (، 97\%) والمستوى الأول (1) محاولة وبنسبة مئوية مقدارها (1)، 9 \%) إما محاولات الضرب الساحق لنادي

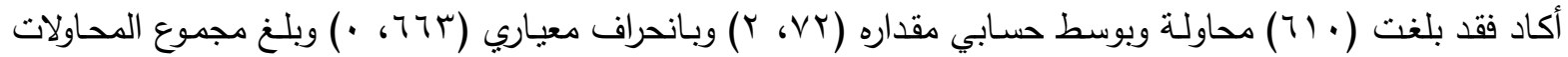

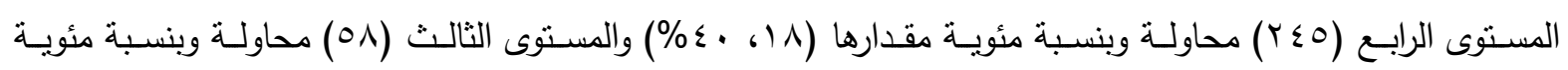

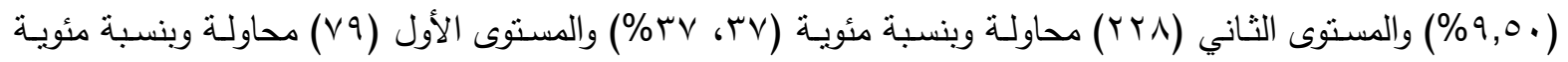

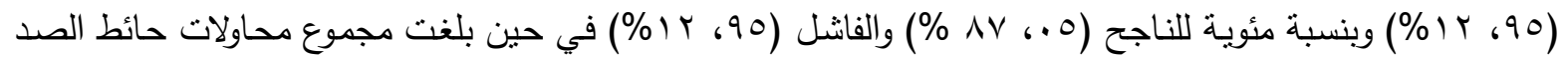

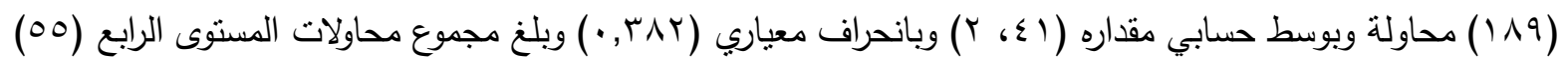

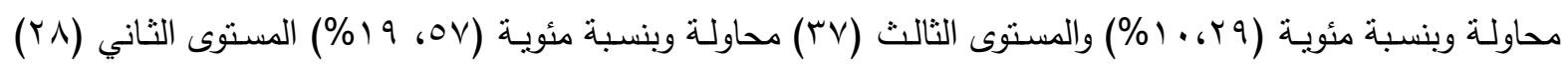

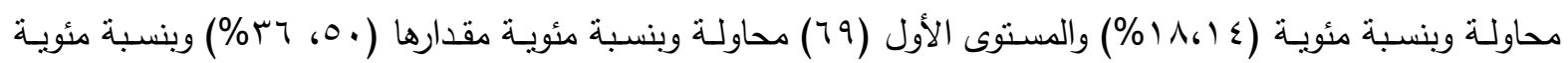

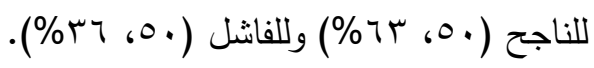

مما تقدم في عرض الجدول (r) نلاحظ الهيمنة العشوائية والتذبذب في أرقام نتائجها ومهارات الدفاعيـة جميعها فلا توجد نتيجة مباراة منتابهة أو تكون مقاربة إلى مباراة ثانية وبالرغم من علو النسبة المئوية للمحاولات الناجحة لكونها لم تصل إلى الدرجة المعنوية ويمكن من خلال ذللك أن المدرب الناجح يستطيع الاعتمـاد على هذه النسب في تقويم المهارات الهجومية للمحاولات الناجحة التي أحرزت نسبة مئوية ناجحة عالية للخروج إلى الجانب المعنوي. وأيضاً نرى النسبة المئوية الضرب الساحق أعلى من المهارات الأخرى ويعزو الباحث وجود لاعبات ذو الخبرة الطويلة في أداء الضرب الساحق وغالبية فريق أكاد لاعبات منتخب الوطني والذي يمتازون بسرعة الحركة والتوقع الصحيح للكرة. وان زيادة العمل معدل حائط الصد لدى فريق أكاد راجع إلى دفاع الفردي لدى بعض لاعبات النادي وخاصة المتقدمين منهم. وأثنارت النقاط المسجلة من جراء حائط الصد واعتماد نادي أكاد على مهارة حائط الصد في محاولات الناجحة بنتيجة مبارياته أي أن الهجوم العشوائي السريع غير المنظم من الفريق المنافس ساعد في الحصول على الكرة من جراء حائط الصد. 
مجلة كلية التربية الرياضية - جامعة بغداد * المجلا السابع والعشرون * العدد الرابع * لسنة

ع - ع عرض نتائج الأوساط الحسابية والانحرافات المعيارية لنادي قرقوش وتحليلها:

الجدول (ع)

يبين الوسط الحسابي والانحراف المعياري لأداء المهارات الهجومية ومجموع المحاولات الكلية لكل مهارة ومستوياتها

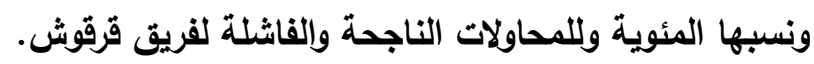

\begin{tabular}{|c|c|c|c|c|c|c|c|c|c|c|}
\hline 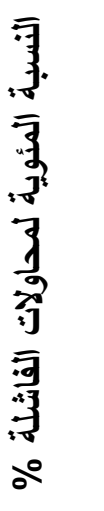 & 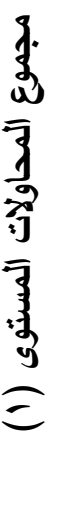 & 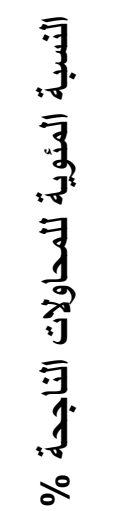 & 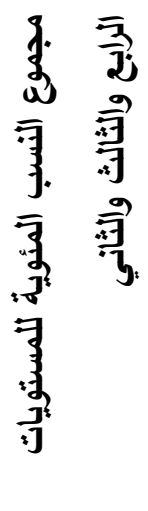 & 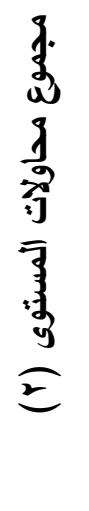 & 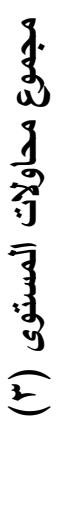 & 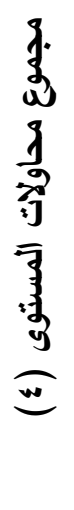 & 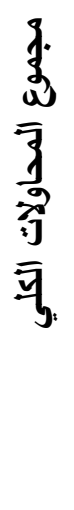 & $\begin{array}{l}\bar{y} \\
\frac{7}{9} \\
\overline{3} \\
3 \\
3:\end{array}$ & $\begin{array}{l}\overline{3} \\
\overline{3} \\
\overline{3} \\
\overline{3}\end{array}$ & $\underset{8}{9}$ \\
\hline$৭, \wedge r$ & $\varepsilon$. & $9 \cdot, 11$ & $\begin{array}{l}+\vee \cdot \wedge\urcorner \\
+|\wedge,| \wedge \\
\nearrow \varepsilon, \mid r\end{array}$ & Y7I & $V \varepsilon$ & rr & $\varepsilon \cdot V$ & or, & $r, I V$ & الإرسال \\
\hline $\begin{array}{l}6 \varepsilon r \\
17\end{array}$ & $\wedge \wedge$ & $\Lambda r, O V$ & $\begin{array}{l}+\varepsilon \vee, q \leq \\
+r, \vee r \\
r, q .\end{array}$ & $|V|$ & $r$. & YOV & דro & $\cdot, \wedge \varepsilon$. & $r, \wedge r$ & الساحق \\
\hline$\{\Lambda, \leqslant 0$ & N1 & 01,00 & $\begin{array}{l}+r \wedge, \circ V \\
+11,1 \wedge \\
11, \wedge .\end{array}$ & 19 & 11 & $\leq 7$ & 171 & $\cdot, \varepsilon \cdot V$ & $r, r)$ & حائط \\
\hline
\end{tabular}

يبين الجدول (ع) الأوسـاط الحسابية والاتحرافات المعياريـة لأداء المهارات الهجوميـة ( الإرسـال، الضـرب

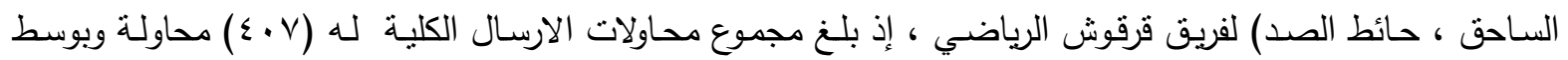

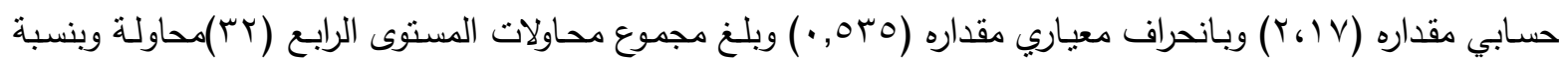

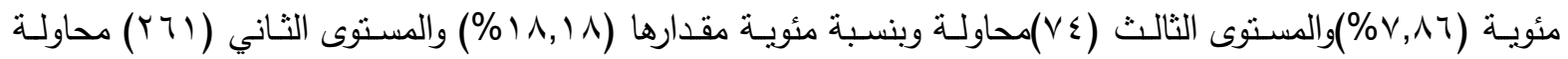

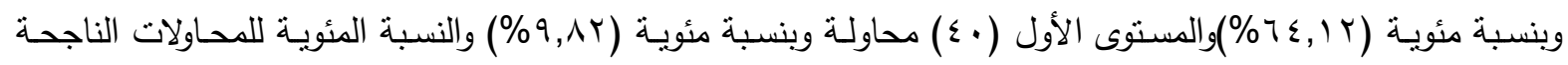

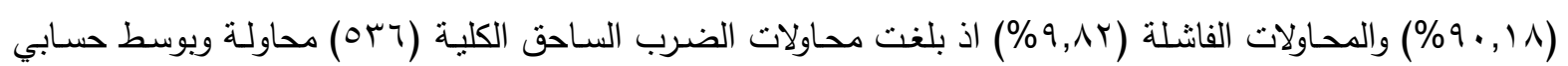

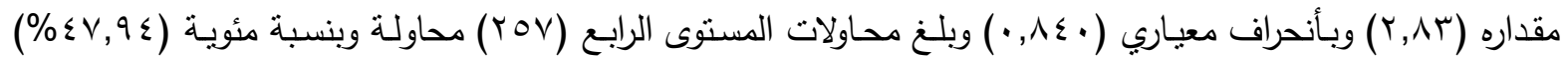




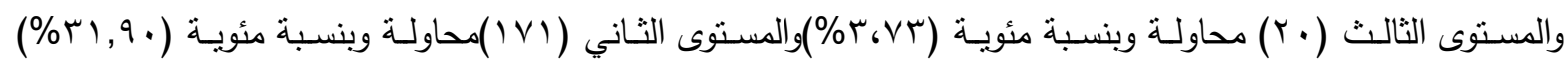

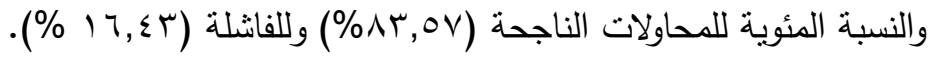

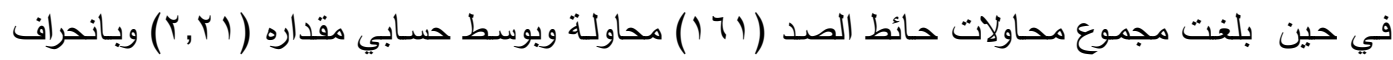

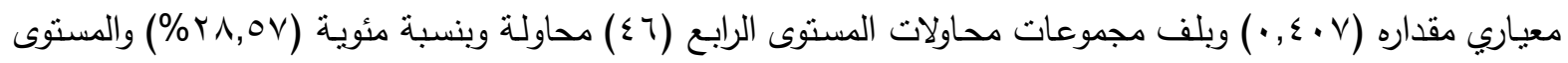

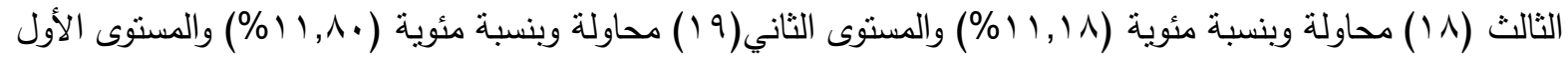

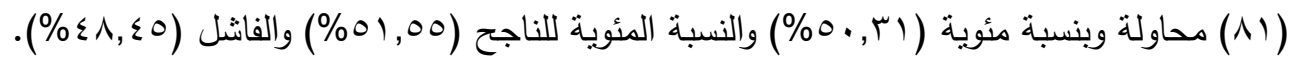

ومن خلال الأرقام المبينة في الجدول رقم (ع) لنادي قرقوش تبين للباحث إن تسجيل النقاط لدى واحد من أفضل أندية العراقية للنساء لايزال يدور في طور عدم الاستقرار والتنبذب وعند الدخول في تفسبر تفاصيل المهارات الهجومية نجد هذا الاختلاف تقل عند حائط الصد الذي لا يدل على حائط الصد متمكن في حين مهارة الارسال كانت نسبة نجاح عالية.

ويعزو الباحث أيضـا إن الفرق العراقية للنساء بصورة عامـة وبغداد بصورة خاصـة يميل إلى التدريب على

$$
\text { الإرسال بصورة صحيحة وهذا يظهر في الجدول (ع). }
$$

وكذللك يلاحظ بنسبة OV ov، للمحاولة نتيجة جيد في الضرب الساحق وكذلك العامل النفسي له أهمية كبيرة في الحفاظ على مستوى اللاعبات أو انخفاضه داخل الملعب وخاصة في فترة المنافسات إذ إن اللاعبات يتعرضن إلى شد عصبي سببه مستوى أداء المباراة" إلا إن الإعداد النفسي يعتبر احد منطلبات الأساسية التي تؤهل اللاعبة لأداء هذه القدرات البدنية بمعدلات ومستويات ثابتة" (11: ب9 ب). فضـلا عن جوانب أخرى تؤثر في مستوى الأداء المهاري للاعبـات داخل الملعب مثل الناحيـة الصحية والتاريخ الرياضي وهذا ويجب الاهتمام بهذه الجوانب باعتبارها مهمة في اللعبة. 
مجلة كلية التربية الرياضية - جامعة بغداد ** المجلا السابع والعشرون * العدد الرابع * لسنة

ع - عرض نتائج الأوساط الحسابية والانحرافات المعيارية لفريق بغداد الرياضي وتحليلها:

الجدول (0)

يبين الأوساط الحسابية والانحرافات المعيارية لأداء المهارات الهجومية ومجموع المحاولات الكلية لكل مهارة مستوياتها والنسبة المئوية والمحاولات الناجحة والفاشلة لفريق الولاء الرياضي.

\begin{tabular}{|c|c|c|c|c|c|c|c|c|c|c|}
\hline 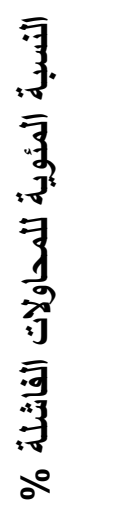 & 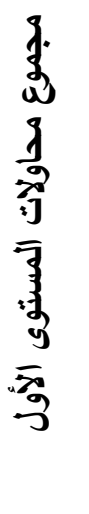 & 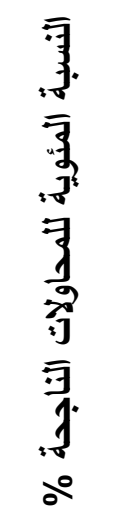 & 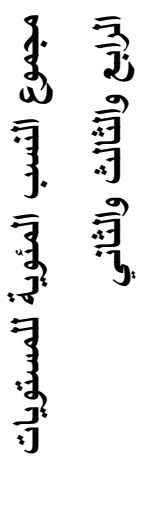 & $\begin{array}{l}\text { j. } \\
3 \\
j \\
3 \\
3 \\
\overline{3} \\
\overline{3} \\
3 \\
3 \\
3 \\
3 \\
3\end{array}$ & $\begin{array}{l}3 . \\
3 \\
3 \\
3 \\
3 \\
\overline{3} \\
3 \\
3 \\
3 \\
\overline{3} \\
\overline{3}\end{array}$ & $\begin{array}{l}\text { j. } \\
3 \\
3 \\
3 \\
3 \\
\overline{3} \\
\overline{3} \\
3 \\
\overline{3} \\
\overline{3}\end{array}$ & $\begin{array}{l}\text { j. } \\
\text { w } \\
\bar{y} \\
\text { jaj } \\
\bar{y}\end{array}$ & $\begin{array}{l}\overline{3} \\
\frac{3}{9} \\
\overline{3} \\
3: \\
3:\end{array}$ & $\begin{array}{l}\overline{3} \\
\overline{3} \\
\overline{7} \\
3 \\
\overline{3}\end{array}$ & 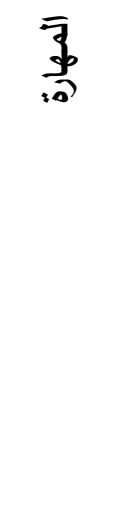 \\
\hline $17, \wedge$. & NT & $\left.\wedge r_{6}\right) 9$ & $\begin{array}{l}+r q, \wedge v \\
+\Sigma, \Sigma \vee \\
r V_{6} \wedge 0\end{array}$ & $11 \mathrm{~V}$ & $r V$ & $19 \mathrm{~V}$ & $\leq 9 \leq$ & $\cdot, 7 \vee 7$ & $\curlyvee, \uparrow \wedge$ & والإرسال \\
\hline$\Lambda, \vee \backslash$ & r & $q 1, Y q$ & $\begin{array}{l}+Y, r \text { O } \\
V_{\cdot, r}\end{array}$ & ro. & $\varepsilon \varepsilon$ & M & rot & $\cdot, 0 \wedge r$ & $r, Y_{I}$ & الضاحق \\
\hline$\leqslant 1, \cdot r$ & $\leqslant 1$ & $0 \Lambda_{6} 9 \mathrm{~V}$ & $\begin{array}{l}+r 96 r 1 \\
+V_{6} .79 \\
111.97\end{array}$ & $1 \varepsilon$ & 9 & $\leqslant 7$ & $11 \mathrm{~V}$ & $\cdot, 7 \leq \varepsilon$ & $r, \leqslant 0$ & الصد حائط \\
\hline
\end{tabular}

يبين الجدول (0) الأوساط الحسابية والانحراف المعيارية لأداء المهارات الهجومية (الارسال، الضرب

الساحق، حائط الصد) لفريق بغداد الرياضي إذ بلغ مجموع المحاولات الإرسال الكلية له (T07) محاولة وبوسط حسابي

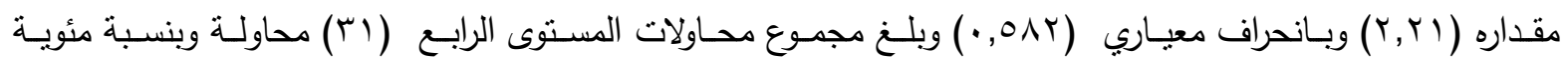

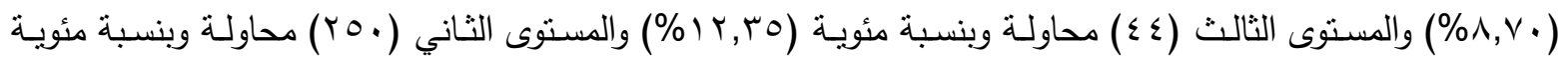

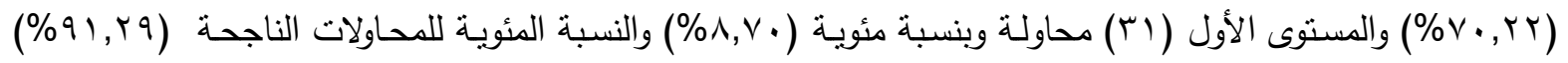

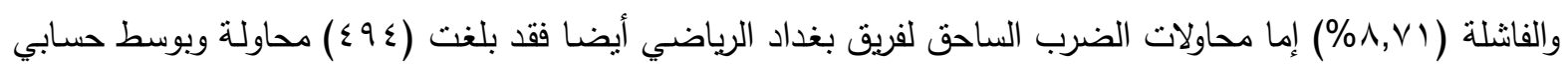

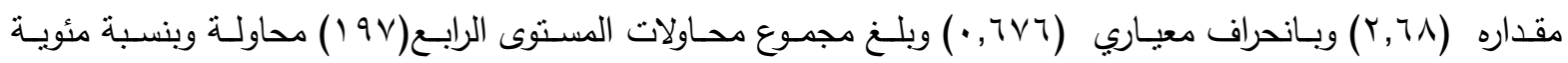




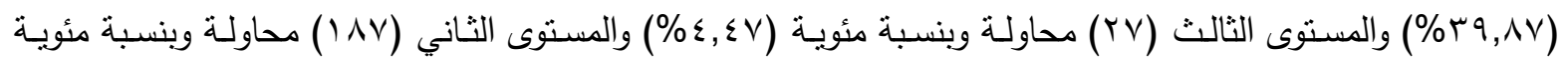

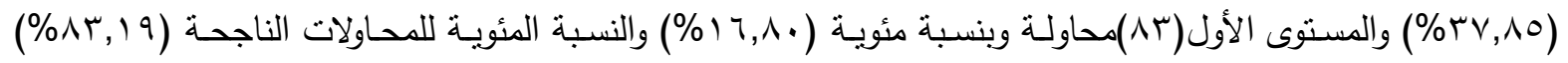

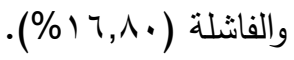

بينما بلغت مجموع محاولات حائط الصد (IVV ) محاولة وبوسط حسابي مقداره (Y, Y) وبانحراف معياري

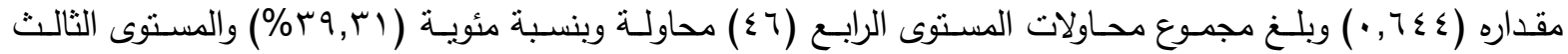

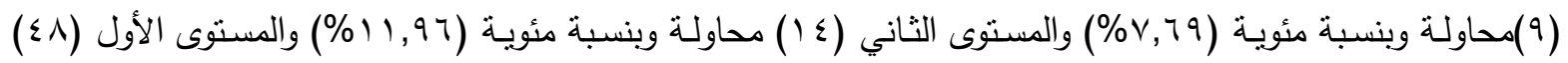

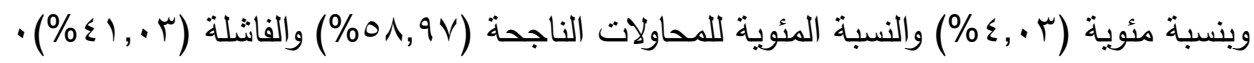

ويعزو الباحث أيضـا إن الفرق العراقية للنساء بصورة عامة وبغداد بصورة خاصـة يميل إلى التدريب على

$$
\text { الإرسال بصورة صحيحة وهذا يظهر في الجدول (0) (ro:I r). }
$$

استتادا الى ارقام نـادي بغداد الرياضي ونتائج مبارياته نلاحظ ان افضل نسبة مئويـة للحساولات الناجحة أثرت لاى هذا الفريق كانت مهارة الضرب الساحق وهذا لان فريق بغداد يمتلك لاعبات شابات جيدات لهن القابلية على اختراق حائط الصد الفريق المنافس ولاعبات الثابات يمتاز في التوقع وسرعة الحركة في الضرب الساحق ونلاحظ في

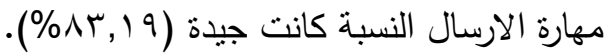


مجلة كلية التربية الرياضية - جامعة بغداد ** المجلا السابع والعشرون * العدد الرابع * للنة

ع - ج عرض نتائج الأوساط الحسابية والانحرافات المعيارية لفريق شقلاوة الرياضي وتحليلها:

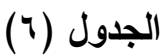

يبين الأوساط الحسابية والانحراف المعيارية لأداء المهارات الهجومية ومجموع المحاولات الكلية لكل مهارة

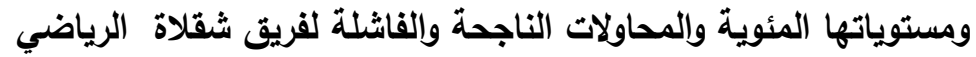

\begin{tabular}{|c|c|c|c|c|c|c|c|c|c|c|}
\hline 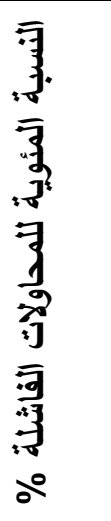 & 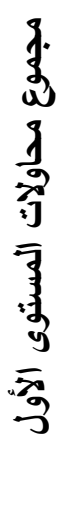 & 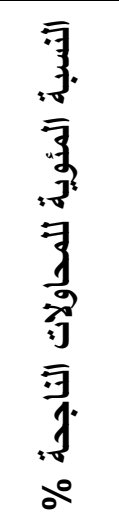 & 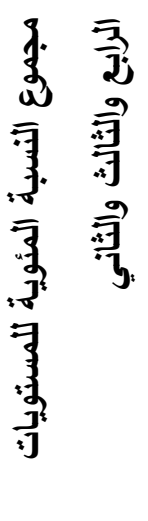 & 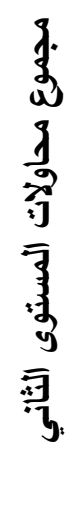 & 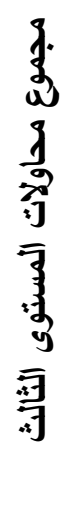 & $\begin{array}{l}\text { j. } \\
3 \\
3 \\
3 \\
3 \\
\overline{3} \\
3 \\
3 \\
\overline{3} \\
\overline{3}\end{array}$ & $\begin{array}{l}\text { z. } \\
\text { a } \\
\overline{3} \\
\overline{3} \\
\overline{3} \\
\overline{\mathbf{y}}\end{array}$ & 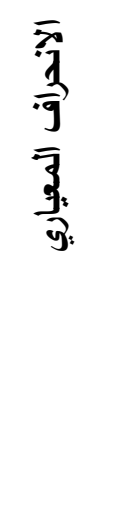 & $\begin{array}{l}\overline{3} \\
\overline{3} \\
\overline{7} \\
3 \\
3\end{array}$ & 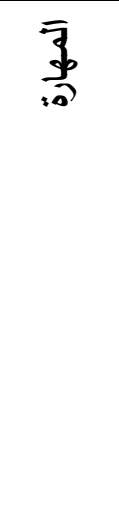 \\
\hline qur & r) & $9 \cdot, 7 V$ & $\begin{array}{l}6+\Lambda, 1 T \\
+\varepsilon, 01 \\
\vee \wedge, \Sigma 1\end{array}$ & roq & 10 & TV & THY & r,00r & $r, I r$ & والإرسال \\
\hline $19,0 \mathrm{r}$ & 9. & $\Lambda \cdot, \leqslant \Lambda$ & $\begin{array}{l}+r 1, \wedge \wedge \\
+r, \wedge 1 \\
\leq 0, \vee V\end{array}$ & YII & 14 & $1 \leqslant V$ & $\{7\}$ & ., $\vee 0$. & $r, V_{0}$ & الضاحق \\
\hline$r v, \cdot r$ & $\varepsilon$. & $7 r, 9 V$ & $\begin{array}{l}+Y q, 7 Y \\
+\mid \wedge, 01 \\
|\varepsilon, \wedge|\end{array}$ & 17 & $r$. & Tr & 1.1 & $\cdot, r q \wedge$ & $r, Y V$ & الصد الصائط \\
\hline
\end{tabular}

يبين جدول (†) الأوسـاط الحسـابية والانحـراف المعياريـة لأداء المهـارات الهجوميـة (الارسـال، الضـرب

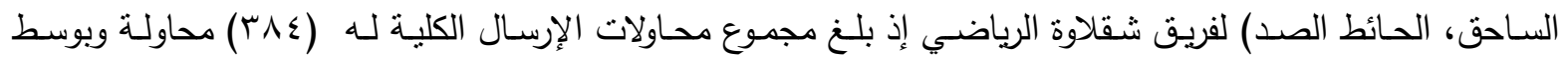

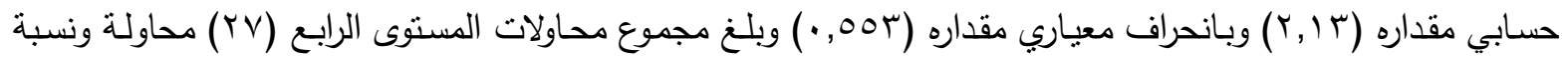

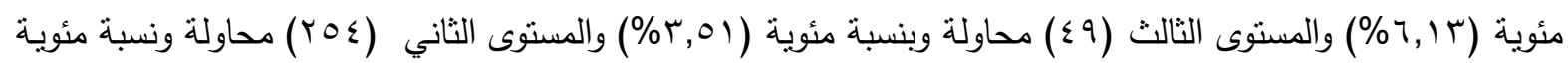

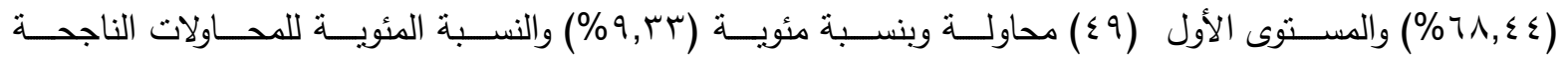

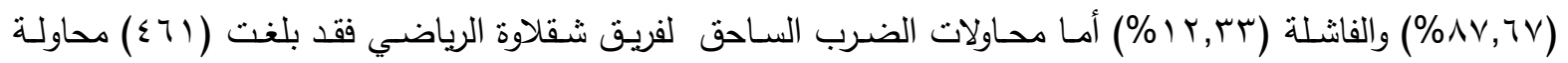

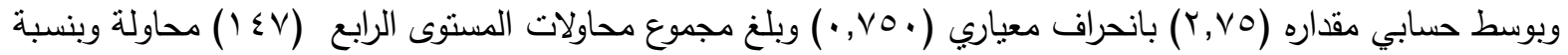

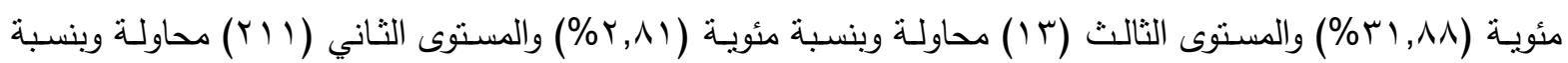


مجلة كلية التربية الرياضية - جامعة بغداد * المجلد السابع والعثرون * العدد الرابع * لسنة

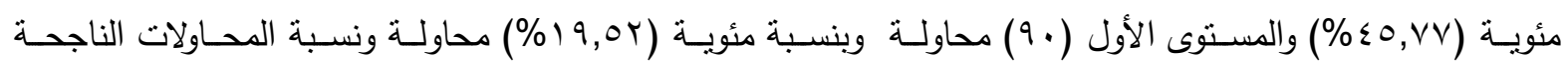

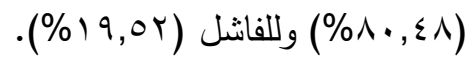

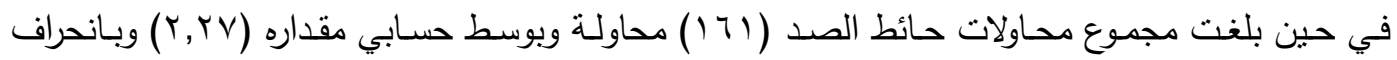

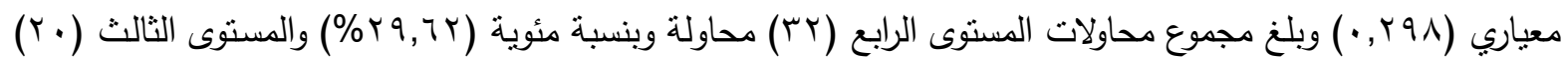

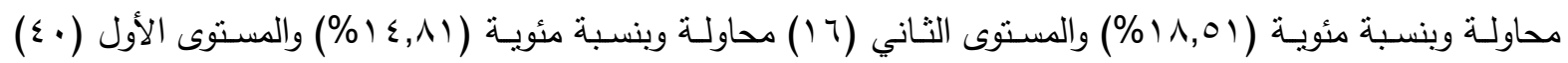

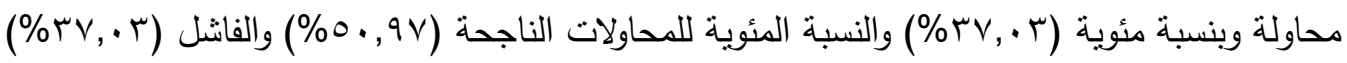

وتعد نتائج فريق نادي شقلاوة في اكبر النتائج نطرفا وعدم الاستقرار واضح في تسجيل النقاط وعلى مدى

المباريات السته وعلى عكس ذلك اظهر استقرار جيد في احد المهارات الهجومية وعلى الرغم وان في أرقامه مقارنهـ بالفرق الثلاثة المشاركة في الدوري.

ويعزو الباحث على إن التدريب غير المبرمج هو السبب في عدم حصول الفريق على مهارات الهجومية

توازي بقية الفرق وبقائه في المرنبة الأخيرة جاء مناسبا لعروضه وأرقامه الإحصائية وان الضعف في مستوى حائط الصد والذي اغلب الاحيان حائط صد فردي وغير منظم وغير جيد (T IVIT).

ويعد نـادي شقلاوة من الفرق التي تمثل الارسال العالي في نسب غير دقيقة في تسجيل النقاط لكرة من المنافس ومن ثم يسجل النقاط استتادا الى النقاط المسجلة من جراء بقية الفرق. إما الضرب الساحق فلم يسهم مساهمة فعالة لأنه لايمتلك لاعبات طوال يجيدون الضرب الساحق وكانت نسبة المئوية للمحاولات الناجحة للفرق الأربعة في الضرب الساحق مسنقرة الحدوث على عكس بقية المهارات الهجومية من هنا اعتمدت على المهارات الهجومية بينها وبين نتيجة المباريات والذي انعكس على نسبه المهارات.

ويرى الباحث إن أفضل أربعة الفرق فريق النساء تسجل نقاطها في الغالب عن طريق الهجوم المنظم سواء

كان عالي أو سريع إحدى المهارات الهجومية تحرز من جرائها النقاط ويثبت رأي الباحث إن اكبر نسبة مئويـة للمهارات الناجحة في النتائج مباريات الفرق الأربعة المشاركة في المربع الذهبي جاء من الضرب الساحق ويأني بعدها الارسال وأخيرا حائط الصد. 
مجلة كلية التربية الرياضية ـ جامعة بغداد * المجلد السابع والعشرون * العدد الرابع * لسنة

ه- المبحث الخامس: الاستنتاجات والتوصيات.

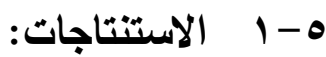

من خـلال النتائج التي توصل إليها الباحث والمعالجة الإحصائية للبيانات والتتغيرات ثم التوصل إلى

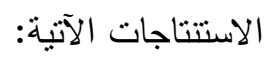

1. ـ هناك عشوائية في الارتباطات لمسنوى أداء المهارات الدفاعية.

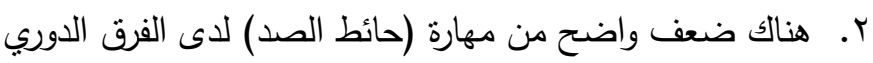

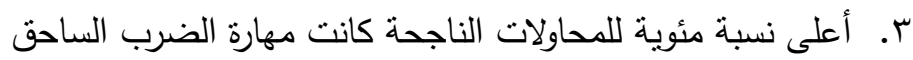

ء. وجود تقارب في مستوى أداء المهارات الهجومية لفرق عينة البحث.

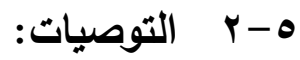

من خلال الاستنتاجات التي توصل إليها الباحث يوصي بما يأتي:

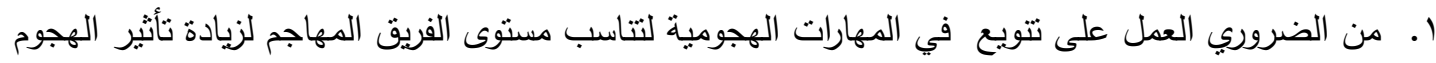

r. التركيز على حائط الصد وإيجاد أكثر من لاعب له القدرة على إتقان مهارة حائط الصد.

r. آ. تخصيص وقت كافي من الزمن الوحدات الندريبية اليومية في المهارات الدفاعية.

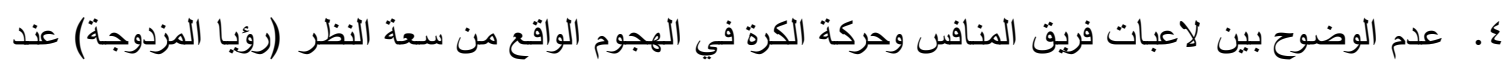

أغلب لاعبات الفرق العراقية من الناحية الهجومية.

ه. ضرورة معرفة العوامل الموئرة على الدهارات الهجومية (زوايا،المسافة ،الارتفاع ،السرعة).

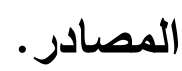

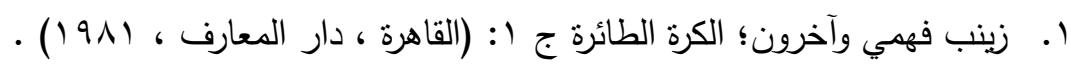

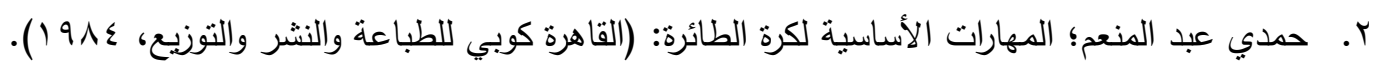
r. عامر جبار؛ مهارة استقبال الإرسال وأثرها في المنهج الهجومي في الكرة الطائرة: (رسالة ماجستير كلية التربية

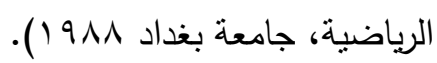

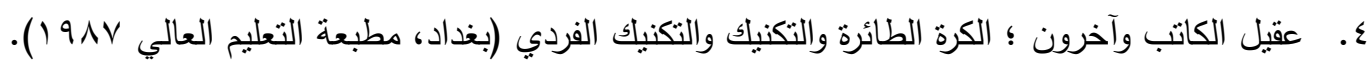
ه. المكتبة الافتراضية ANAL YSIS OF BEACH VOLLEYBALL ACTION SEQUENCES OF FEMALE TOP ATHL ETES: Koch, Christina. Tilp , Markus. Analysis of beach

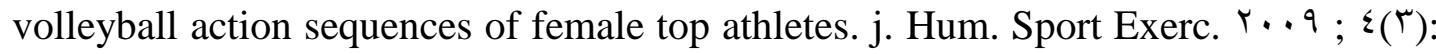


مجلة كلية التربية الرياضية - جامعة بغداد * المجلد السابع والعثرون * العدد الرابع * لسنة

7. وجيه محجوب؛ اصول البحث العلمي ومناهجه: (عمان، دار المناهج للنشر والتوزيع، 999 (1) ) V. نوري إبراهيم الثوك ورافع صالح فتحي الكبيسي ؛ دليل البحث لكتابة الأبحاث في التربية الرياضية: (بغداد،

$(r \cdot \mathcal{L}$

^. مصطفى حسين ناجي، المعلومات العلمية بين النظرية و التطبيق، (القاهرة، مركز الكتاب للنشر، 999 ( ) 9. نزار الطالب ومحمود السامرائي ، مبادئ الإحصـاء والاختبارات اليوميـة والرياضية: (الموصل ، مؤسسـة دار الكتب للطباعة والنشر ، (91 (1)).

• 1. قاسم حسن المندلاوي وآخرون، الاختيارات والقياس والتقويم في التربية الرياضية: (بغداد، مطبعة التعليم العالي، .71 ص (1911

الـ محمد صبحي حسنين وحمدي عبد المنعم، الأسس العلمية للكرة الطائرة وطرق القياس، طا، القاهرة، مطبعة

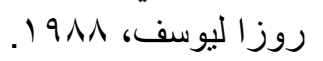
rا ـ علي سبهان؛ التوفع وسر عة الاستجابة الحركيـة و علاقتها بدقة أداء المهار ات الدفاعيـة للاعب الحر بـالكرة

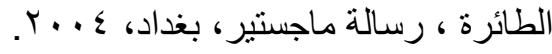

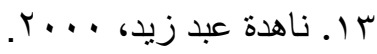

\section{ملحق رقم (1)}

الخبراء اللأين عرض عليه الاستمارة.

$$
\begin{aligned}
& \text { كلية التربية الرياضية - جامعة بغداد. } \\
& \text { كلية التربية الرياضية - جامعة المستتصرية. } \\
& \text { أستاذ } \\
& \text { استاذ مساعد جامعة بغداد - كلية التربية الرياضية. } \\
& \text { طالب دكتوراه جامعة بابل. } \\
& \text { مدرب المنتخب العراقي سابقاً . مابل } \\
& \text { مدرب المنتخب الثباب للمعوقين. }
\end{aligned}
$$

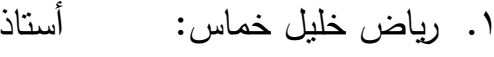
r. r. عسين سيهان ع. علي سيهان

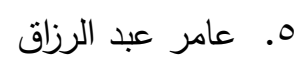
T. حسين عدنان

\section{ملحق رقم (ץ) - (ץ)}

\section{الخبراء}

كلية التربية الرياضية - جامعة بغداد.

مدرب دولي بكرة الطائرة ا. أ. د. علي يوسف كلية التربية الرياضية - جامعة بغداد. لاعب ومدرب منتخب العراقي سابقاً r. أ. د. طارق حسن رزوقي جامعة التكنولوجيا. لاعب ومدرب منتخب العراقي سابقاً r. ا. بل علي عزيز 